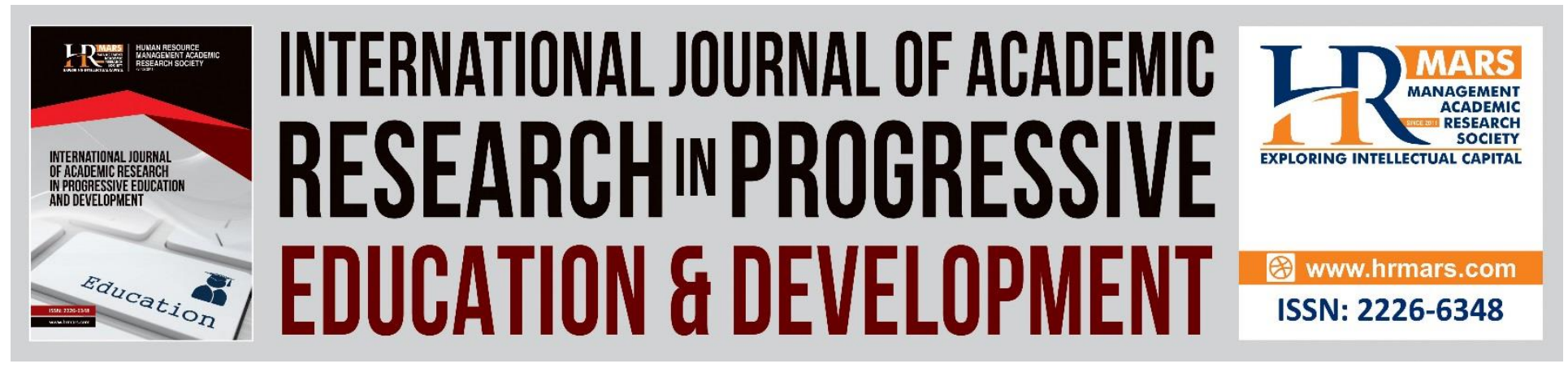

\title{
Kawaii-Style Pedagogical Agents Designs in Virtual Learning Environment: A Research Conceptual Framework
}

Kaliyamah Raman, Ahmad Nizam Othman, Muhammad Zaffwan Idris, Vicneas Muniady

To Link this Article: http://dx.doi.org/10.6007/IJARPED/v10-i1/8489 DOI:10.6007/IJARPED/v10-i1/8489

Received: 03 December 2020, Revised: 01 January 2021, Accepted: 17 January 2021

Published Online: 30 January 2021

In-Text Citation: (Kaliyamah et al., 2021)

To Cite this Article: Kaliyamah, R., Ahmad Nizam, O., Muhammad Zaffwan, I., \& Vicneas, M. (2021). Kawaii-Style Pedagogical Agents Designs in Virtual Learning Environment: A Research Conceptual Framework. International Journal of Academic Research in Progressive Education and Development, 10(1), $154-170$.

Copyright: (C) 2021 The Author(s)

Published by Human Resource Management Academic Research Society (www.hrmars.com)

This article is published under the Creative Commons Attribution (CC BY 4.0) license. Anyone may reproduce, distribute, translate and create derivative works of this article (for both commercial and non-commercial purposes), subject to full attribution to the original publication and authors. The full terms of this license may be seen at: http://creativecommons.org/licences/by/4.0/legalcode

Vol. 10(1) 2021, Pg. 154 - 170

http://hrmars.com/index.php/pages/detail/IJARPED JOURNAL HOMEPAGE

Full Terms \& Conditions of access and use can be found at http://hrmars.com/index.php/pages/detail/publication-ethics 


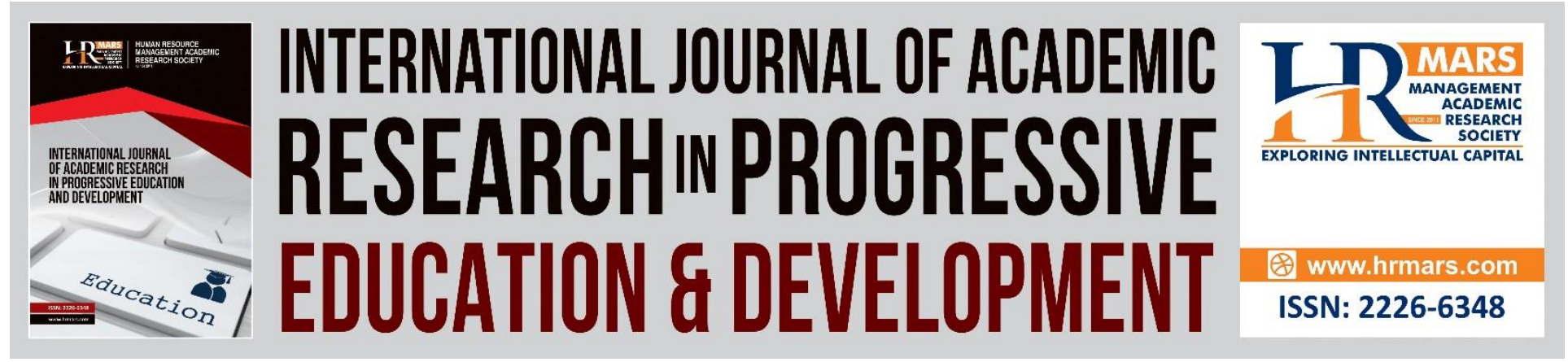

\title{
Kawaii-Style Pedagogical Agents Designs in Virtual Learning Environment: A Research Conceptual Framework
}

\author{
Kaliyamah Raman ${ }^{1}$, Ahmad Nizam Othman ${ }^{1}$, Muhammad Zaffwan \\ Idris $^{1}$, Vicneas Muniady ${ }^{2}$ \\ ${ }^{1}$ Faculty of Art, Computing and Creative Industry, Universiti Pendidikan Sultan Idris, 35900 \\ Tanjong Malim, Perak, Malaysia, ${ }^{2}$ Department of Information and Communication Technology, \\ Politeknik Sultan Azlan Shah, 35950 Behrang Steysen, Perak, Malaysia \\ Email: logesri02@gmail.com
}

\begin{abstract}
Pedagogical agents are animated virtual characters that play human instructional roles in educational settings. Towards the advancement of computer technology and communication, human learning is becoming more advanced by using technologies such as pedagogical agent. Previously, the look of pedagogical agents has been designed with own imagination of the designers without assessing its impact on the learning. In addition, there are limited researches on the visual appearance of pedagogical agent. Therefore, a further study is required to fill this gap and provide appropriate visual appearance for pedagogical agent that will contribute to enhancing students' motivation in learning. Accordingly, the present research proposes kawaii designs for the facial appearance of pedagogical agent. Eventually, students' affective state on the kawaii style pedagogical agents and relation towards motivation in learning also will be studied. Therefore, this paper proposes a conceptual framework that acts as guidelines in the development of kawaii style pedagogical agents to increase students' motivation in virtual learning environment. Heidig \& Clarebout's PALD model, Keller's ARCS model of motivational design, Nittono's two-layer model of kawaii and Watson \& Tellegen's Circumplex model of affect are used as a basis to construct conceptual framework in this study.
\end{abstract}

Keywords: Baby Schema, Conceptual Framework, Cute, Kawaii, Pedagogical Agent, Virtual Learning Environment

\section{Introduction}

A pedagogical agent is an animated lifelike character with human-like behaviours, speech, gesture and movement that used for computer-aided learning (Park, 2018). Pedagogical agents are widely implemented in various virtual learning platforms. Accordingly, pedagogical agents serve various instructional roles such as mentor (Baylor and Kim, 2005), learning companion 
(Johnson and Lester, 2016), tutors, coaches, life-long learning partners (Mohanty, 2016) and teachable agents (Biswas et al., 2004).

In instructional settings, pedagogical agent can represent a human role through effective communication with students in both visually and verbally (Vicneas and Zamzuri, 2019; Vicneas and Zamzuri, 2020). Adding such animated pedagogical agent in Virtual Learning Environment (VLE) creates social connection between pedagogical agent and students that turns to promote interest in learning task among students (Goldberg and Cannon-Bowers, 2015). It seems clear, during the social interaction between pedagogical agent and students, physical cues of the pedagogical agent profoundly affect students' belief and their behaviour (Gulz and Haake, 2006). In view of that, a well-designed animated pedagogical agent in educational context is potential to increase motivation among students (Dale, 2016). Along these lines, greater importance ought to be given on the design of pedagogical agent.

Interestingly, cuteness is becoming a 'dominant aesthetic of the digital culture and consumer culture of the current century'. In fact, the trend of cuteness is a blooming popularity in all things, products and so on. The existence of cuteness can be clearly seen in modern Japanese aesthetic of kawaii which is widely adapted and recognized among people now (Sloan, 2015). On the other hand, kawaii aesthetics have stretched beyond the borders of Japan and today it has been practised in numerous countries to manufacture various products. As kawaii is being treated favourably, it is capable to induce positive affective state and consequently influences the subsequent behaviour of the people (Sherman et al., 2009; Nittono et al., 2012a). Moreover, the idea of kawaii can be expanded with the baby schema theory. According to Lorenz (1943), any object with certain facial features such as high forehead, big eyes, an oversized head, and full cheeks are capable to trigger positive affective feelings among viewers (Lieber-Milo and Nittono, 2019). Animated kawaii characters which comprise visual characteristics of baby schema are obtained vast popularity. Consequently, characters like Hello Kitty, Pokemon and Doraemon obtained million-dollar commercial success. Surprisingly, the characters affect millions of viewers around the world. The worldwide success of these characters as a consequence of emotional reaction in humans against the power of cuteness (Marcus et al., 2017).

Therefore, question arises how the students' affective state would be if different kawaii designs are adapted in the design of pedagogical agent for education settings. Another question is to find out whether the different designs of kawaii affect students' motivation in learning. Therefore, this paper proposes a conceptual framework as the guidelines in developing kawaii style pedagogical agents in VLE. The adaptation of kawaii style in the design of pedagogical agent is a novel idea where it may affect students positively in VLE as well. Therefore, this research is conducted to fill this gap.

\section{Theoretical Background}

\section{a. Pedagogical Agent}

Animated virtual characters are virtual life-like characters that play roles as good conversational partners in animations, movies, and cartoons (Liu et al. 2019). In a same way, pedagogical agents are animated virtual characters that play human instructional roles in educational settings (Vicneas and Zamzuri, 2019; Vicneas and Zamzuri, 2020). Towards the advancement of computer technology and communication, human learning is becoming more advanced by using 
technologies such as pedagogical agents (Baylor and Kim, 2005). Pedagogical agents widely used in various teaching and learning environment with many different roles such as mentors, motivators, facilitators, navigators, and collaboration assistants (Martha and Santoso, 2019).

A pedagogical agent that rendered on a computer screen is capable to facilitate learning of the presented material (Heidig and Clarebout, 2011) and guide the students throughout the learning process (Clark and Mayer, 2003). In addition, pedagogical agents can also provide support and feedback to the students (Dinçer and Doğanay, 2017). It seems clear that pedagogical agents play a very important role in their social interactions with students.

When an appealing pedagogical agent is presented in VLE, students are capable to practise student-centered learning and they would emotionally connect to the agent (Dinçer and Doğanay, 2017). Hence, a strong bond is formed between students and pedagogical agent that is desirable to them. Students' affective responses are capable to recognize their moods towards pedagogical agents that are being presented to them such as happy, confused, frustrated, surprised or bored (Mohanty, 2016). The identification of these positive and negative affective state is very important to speed up learning process in positive way in the VLE (Kort et al., 2001). In view of that, visual appearance of pedagogical agent is a vital design element to capture students' attention and establish their first impression and subsequently trigger students' affective state as an important cue to identify the effectiveness and efficiency of a learning environment (Mohanty, 2016).

Moreover, pedagogical agents are fascinating guides which can lead in increase motivation and allow students to maximise the learning materials (Dinçer and Doğanay, 2017). Students who exposed to pedagogical agent demonstrated higher motivation and deeper learning than students without an agent (Kim and Baylor, 2006). There are three effects of the increment of motivation in learning (Gulz and Haake, 2006). Firstly, motivation affect students' participation in learning process. Secondly, motivation affects the quality of attention of the students on the learning environment. Thirdly, motivation affects the organization of students' memory. In other words, the use of animated pedagogical agent in VLE engages students to the point that it attracts students' attention to such an extent that improve their retention in learning process.

Consequently, students' motivation increased positively when pedagogical agent looks similarly like students (Shiban et al. 2015). Students tend to prefer pedagogical agent with same age group with them (Johnson et al. 2013). Moreover, learning and motivation are interrelated and it can be enhanced by using the pedagogical agent of the same age (Vicneas and Zamzuri, 2019; Vicneas and Zamzuri, 2020). This is in line with the capability of human-like pedagogical agent to ensure students' interactions much smoother for various teaching and learning functions. Pedagogical agent with actual human beings' pictures is the exceptional pedagogical agent types (Rodicio and Sánchez, 2012). Generally, viewers developed more positive attitudes on human-like agent in contrast to robot agent (Li et al. 2016). However, the pedagogical agent with too realistic look will cause the students uncomfortable (Vicneas and Zamzuri, 2019; Vicneas and Zamzuri, 2020). In this case, determining how to design an appealing human-like pedagogical agent that look similarly like students and make it impressive to them as target audience is an important part in VLE. 


\section{b. Kawaii}

It is believed that rich and interesting facial look of pedagogical agent has a powerful ability to make learning more fun. In view of that, face is a primary tool for interaction that act as mirror to reflect identities and it is foremost aspect of pedagogical agent's design (Withrow and Danner, 2007). The first look at the appearance of the face able to stimulate affective responses among people (Miesler et al. 2011). Notably, the "cute" looking face is very essential as audience identifies with it the most. The term of "cute" reminds of the famous Japanese culture which is called as kawaii. The most popular word of kawaii in Japan means cute in English (Nittono et al. 2012; Takamatsu, 2018). Besides bringing the mean of cute, kawaii also emphasis on the qualities of being sweet, adorable, innocent, and vulnerable in the physical appearances (Wong, 2017). Cuteness is a powerful motivational force that induces states of readiness to engage in social affiliation (Takamatsu, 2018). Nittono (2016) stated that the cuteness in kawaii style related to human sociality that motivates people to interact socially with the given character by priming affiliative, friendly tendencies and imbuing mental states in the entity'.

Kawaii prevailed in inanimate objects such as desserts, fashion accessories, home decorations as well as baby animals and cartoon characters (Nittono and Ihara, 2017). The kawaii design has travelled outside of Japan and influenced world-wide consumer cultural phenomenon through the production of various products with kawaii aesthetics. The kawaii culture also can be seen in Ramayana, Sanskrit epics of ancient India where the character of Hanuman (human/monkey) carried all cute characteristics. The kawaii characteristics of Baby Hanuman's design (figure 1) has taken Indian fans all over the world by a storm (Cheok and Fernando, 2012).

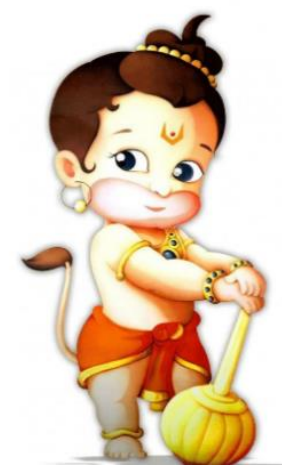

Figure 1: Baby Hanuman (Return of Hanuman, 2007)

Besides that, brands such as Mini, Motorola and Volkswagen have adopted cute as design aesthetic and successfully produced a variety of products that received best response from people around the world (Granot et al., 2014). From there, question arises how if the same effects will befall in educational settings when pedagogical agent designed in kawaii style? It is a research interest to identify students' affective state on kawaii design of pedagogical agent. 


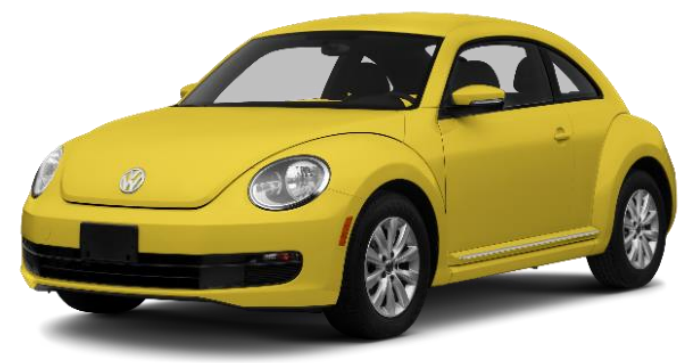

Figure 2: Volkswagen New Beetle (Volkswagen New Beetle, 2013)

\section{c. Baby Schema}

Often, the famous Japanese culture of kawaii is characterized by baby schema. In particular, infantile features such as large curved forehead, big eyes, a pug nose, round cheeks, and small chin are definite as a sign of the baby schema (Takamatsu, 2018). Numerous studies have proposed that infant faces competent to catch viewers' attention at the beginning phase of visual processing (Nittono, 2016). This has been emphasized in the baby-schema concept where it reflects the cuteness of an object. Living things with the features of baby schema perceived as cute and tend to receive approach from other individuals (Nittono, 2010). At the same time, there are various kind of kawaii characters with baby schema that produced and exported to many countries such Pokémon, Doremon, Hello Kitty and so on.

Pokémon is a kawaii character with baby schema that successfully reached global popularity through the television show, computer games and artificial products such as Pokémon dolls (Sherman et al. 2009). Adding to this, worldwide interest has been boosted in the Pokémon with the release of Pokémon Detective Pikachu movie in 2019 with a worldwide gross of over $\$ 431$ million (Sherman et al. 2009). The movie is the eleventh highest grossing film of 2019 and the second highest-grossing video game film adaptation of all time (Detective Pikachu, 2020). Moreover, the design of Pokémon Detective Pikachu in the movie is likable to the audience and praised by the movie reviewers as shown in the figure 4.

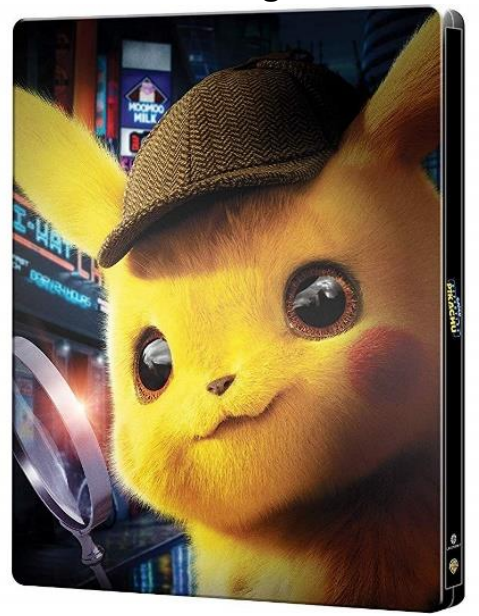

Figure 3: Pokémon Detective Pikachu (Detective Pikachu, 2020) 


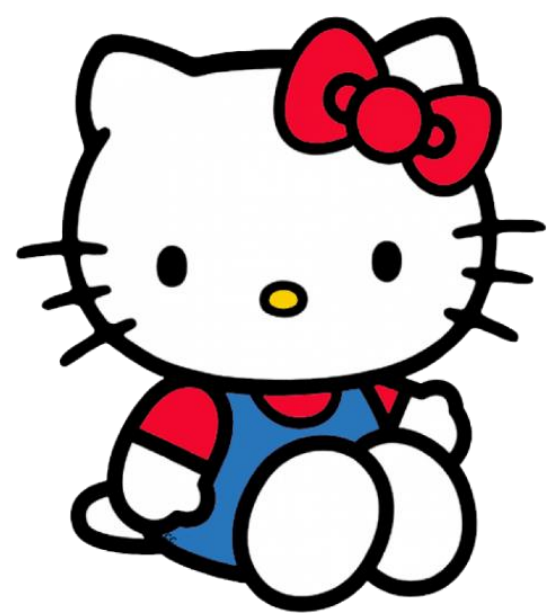

Figure 4: Hello Kitty (Hello Kitty, 2014)

At the same time, Hello Kitty achieved a global success where it has been penetrated in the international market (Pellitteri, 2018). Sanrio company that producing Hello Kitty (figure 4) reaps in approximately US\$3 billion annually (Granot et al. 2014). It seems clear that kawaii characters with baby schema have affected the audience and leaving a lasting impression among them. The characteristic of kawaii with baby schema features are the key factor for the success of a cartoon or an animation production. From that point of view, question arise whether the same success can be achieved in education field if the pedagogical agent that used in VLE is designed in kawaii style with baby schema features?

In conclusion, present study fills the gap through the development of different kawaii designs for pedagogical agents to exhibit appealing visual appearance with the ability to trigger students' affective state and consequently enhance their motivation in learning.

\section{Theoretical Framework}

Pedagogical agents widely implemented to inspire students participate actively in multimedia learning environment. Appealing design of pedagogical agent is essential to raise students' interest and boost their affective state. However, the design of pedagogical agent continues to be complicated. The concern on how to design a pedagogical agent is great importance in the study about pedagogical agent. As for the detailed design of a pedagogical agent, there are many factors that need to be taken into consideration.

Therefore, the Heidig \& Clarebout's Pedagogical Agents-Levels of Design (PALD) model (Heidig and Clarebout, 2011), Keller's ARCS model of motivational design (Keller, 1983), Nittono's two-layer model of kawaii (Nittono, 2016) and Watson \& Tellegen's Circumplex model of affect (Keller, 1983) are used as the basis to construct conceptual framework in this study.

\section{a. Pedagogical Agents-Levels of Design (PALD)Model}

A well-designed pedagogical agent in a VLE can result in successful learning experiences. In accordance with this, Heidig and Clarebout (2011) introduced a model as a framework to design a pedagogical agent which is known as Pedagogical Agents-Levels of Design (PALD) model (figure5). The application of a coherent design process in the PALD model is very important to 


\section{INTERNATIONAL JOURNAL OF ACADEMIC RESEARCH IN PROGRESSIVE EDUCATION AND}

DEVELOPMENT

Vol. 10, No. 1, 2021, E-ISSN: 2226-6348 @ 2021 HRMARS

justify the pedagogical agent's visual appearance. There are three important levels in the PALD model namely, global level, medium level, and detail level. Based on the global level, pedagogical agent can be represented as a non-human or a human to interact with the content area in which agents function to influence learning. In a medium level, the technical decisions such as animation level, lifelikeness, partial presentation, realism, speech style and voice output are determined. Finally, characteristics that need to be considered in detail level are age, gender, clothing, weight and so on. Overall, the consideration about the visual appearance of a pedagogical agent based on PALD model is very essential as it reflects an identity to students (Sloan, 2015).

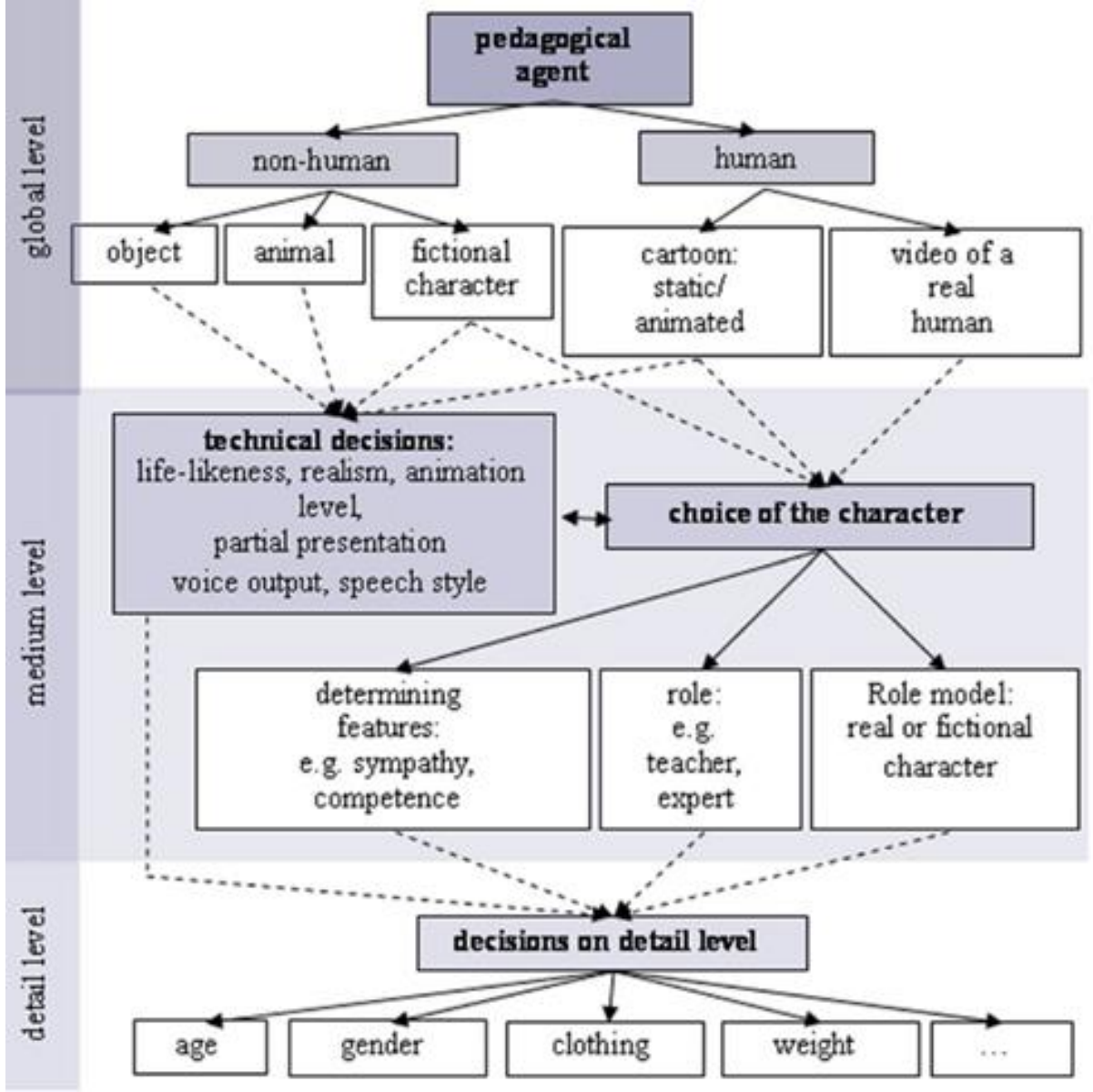

Figure 5: Pedagogical Agents Levels of Design (PALD) Model (Rodicio and Sánchez, 2012)

\section{b. Keller's ARCS Model of Motivational Design}

The VLE is developed based on the Keller's ARCS Model of Motivational Design (1983) as shown in figure 6. The ARCS contains four main categories which are attention, relevance, confidence, and satisfaction (Khan et al. 2019). It is imperative to motivate students and ensure the coherence of the motivation remains throughout the learning in VLE.

Accordingly, the VLE will be presented in an interactive way by adding an attractive animated pedagogical agent to deliver the learning content clearly, interesting navigation control buttons and accessibility interface design of screens to catch the attention of students in ensuring they stay focus on the digital learning content. Basically, an effective way to improve the learning 
Vol. 10, No. 1, 2021, E-ISSN: 2226-6348 @ 2021 HRMARS

and retention is to enhance the interaction between the lecturer and students (Hollands and Tirthali, 2014). Thus, one of the strategy for improving interactivity in VLE is to use pedagogical agent. The animated pedagogical agent is a main feature in the VLE that plays a role as a guide on the side to encourage self-learning by the students. The explanatory of the pedagogical agent comes with visualization that use multimedia elements such as text, graphic and simple movement of animation. Within the content of VLE, students must listen carefully, understand the knowledge and evaluate their knowledge which can be done through quiz session. When the VLE serves as an effective learning method, students would be able to better comprehend to the learning content. Therefore, this model is fundamental to develop the VLE, especially the pedagogical agents.

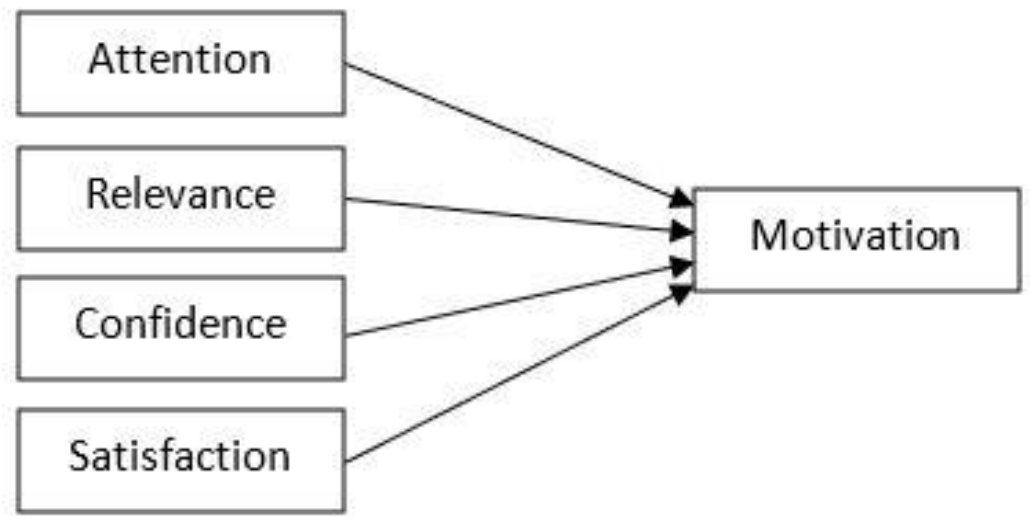

Figure 6: Keller's ARCS model of motivational design (Keller, 1983)

\section{c. Nittono's two-layer model of kawaii}

Aside from that, the visual appearance of a pedagogical agent serves as the most vital aspect of designing that determines the effectiveness of the agent (Yu, 2015). The appealing visual appearance of pedagogical agent is directly related to the stimulation of affective state and in turn promote motivation in learning. In view of that, cuteness can be described as an appealing physical visual appearance that engage social behaviours including companionship, cooperative action, and communication through affective attributes (Dale, 2016). The cuteness of kawaii style with the features of youthfulness and childlikeness is a popular aesthetic style in Japanese media among all age (Occhi, 2012). Basically, kawaii can be defined as affective individual experience (Nittono, 2010). In 2016, Nittono introduced a two-layer model of kawaii (figure 7). The two-layer model of kawaii is a framework to understand about kawaii from a behavioural science perspective. According to Takamatsu (2018), 'the two-layer model of kawaii treats the baby schema as a multi-faceted construct and extends it by adding to Japanese concepts of kawaii' (Takamatsu, 2018).

According to Nittono (2016), 'kawaii is viewed as a psychological state indirectly induced by the perception of certain attributes of a stimulus and the cognitive appraisal of the relationship between the person and the stimulus'. In other words, this model postulates that the basis of kawaii linked to social motivation for engaging and continuing with more desirable persons or objects as an fondness towards baby-schema. Baby schema is one of the central 
element that induce the moods of kawaii (Nittono, 2016). In addition, other attributes such as smile, roundness and colour are powerful factor that may elicit the feelings of kawaii.

In this model, kawaii viewed as emotion which can be characterized as positive, unthreatened, moderately aroused, approach-motivated, and socially oriented. Kawaii has three characteristics which are subjective feelings, behaviour, and physiology. Accordingly, it is important to develop different kawaii designs of pedagogical agent to identify the level of affective state among students. In view of that, the two-layer model of kawaii also used as a guideline to develop conceptual framework for the present study.

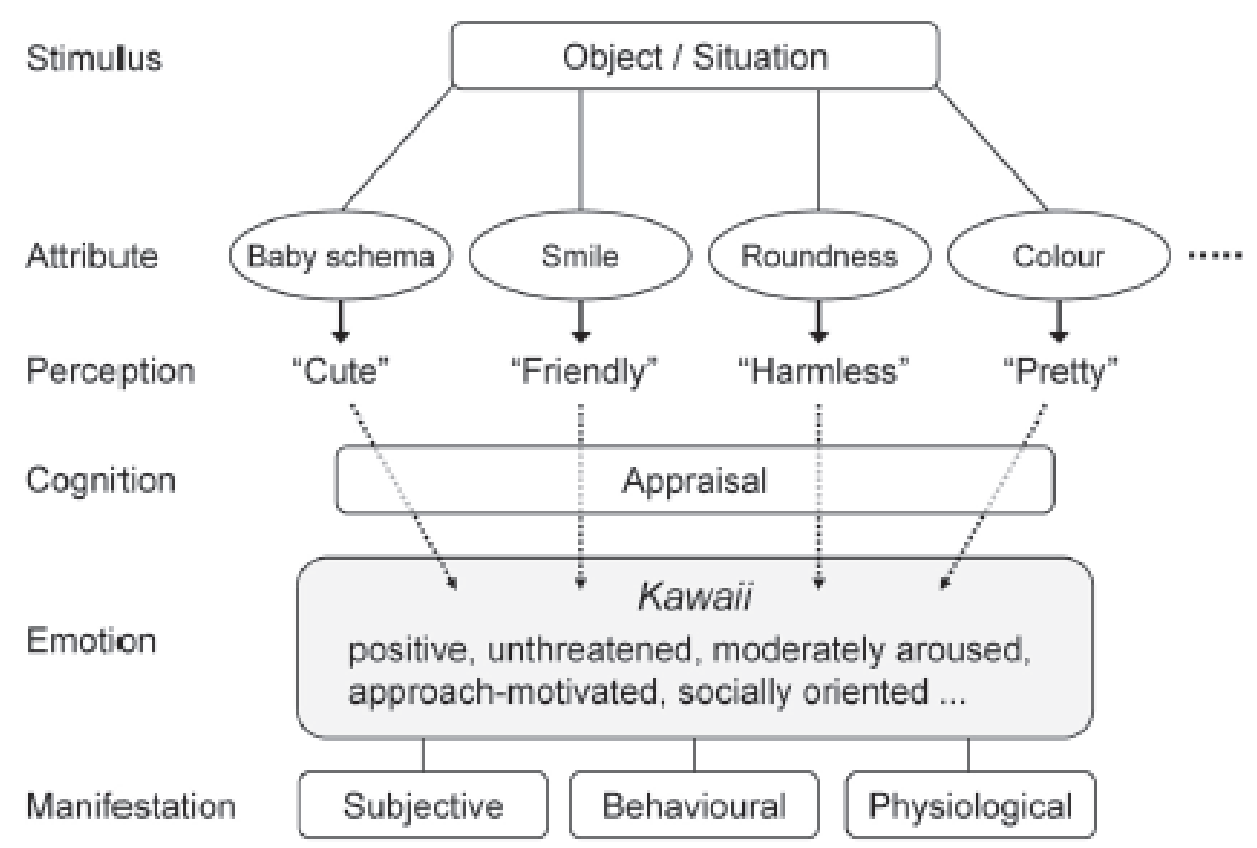

Figure 7: Two-layer model of kawaii (Nittono, 2016)

\section{d. Watson \& Tellegen's Circumplex model of affect}

Meanwhile, the Circumplex Model of Affect by Watson \& Tellegen in 1985 is also used in constructing conceptual framework in this study as shown in figure 8 . The circumplex model of affect based on two dimensions which are known as positive and negative affects. Both positive and negative affects occur on bipolar continua, ranging from high to low (high and low positive affect and high and low negative affect) which result in various moods. Positive affect and negative affect are independent orthogonal dimensions that not at opposite ends of the same continuum (Quinn, 2015). The circumplex model of affect emphasized the importance of the positive affect and negative affect that are depicted by the solid lines. Negative affect on the horizontal axis ranges from low negative affect to high negative affect whereas positive affect on the vertical axis ranges from low positive affect to high positive affect.

There are also two further dimensions which called as pleasantness and engagement. It is combination of positive and negative affects. Combination of high positive affect and low negative affect causes pleasantness. While combination of low positive affect and low negative 
affect causes disengagement. In other words, there are two pairs of dimensions in circumplex model of affect. The first pair consists of high positive affect against low positive affect and high negative affect against low negative affect. The second pair consists of the Pleasantness-VersusUnpleasantness and Engagement-Versus-Disengagement dimensions.

Particularly, people's interest and enjoyment on current task were increases by positive affect (Isen and Reeve, 2005) that elicit them to give maximum of focus (Antunes et al., 2019). From there, it increases the inherent satisfaction that they feel from the task (Antunes et al., 2019). Obviously, it appears capable of increasing intrinsic motivation. In other words, positive affect capable of triggering intrinsic motivation to do a task (Peñalver et al. 2019). Beside motivation, positive affect has been linked to performance, achievement, creativity, and effort on future oriented tasks (Tenney et al. 2016). Surprisingly, kawaii described as a positive affect with strong approach of motivation (Nittono, 2016).

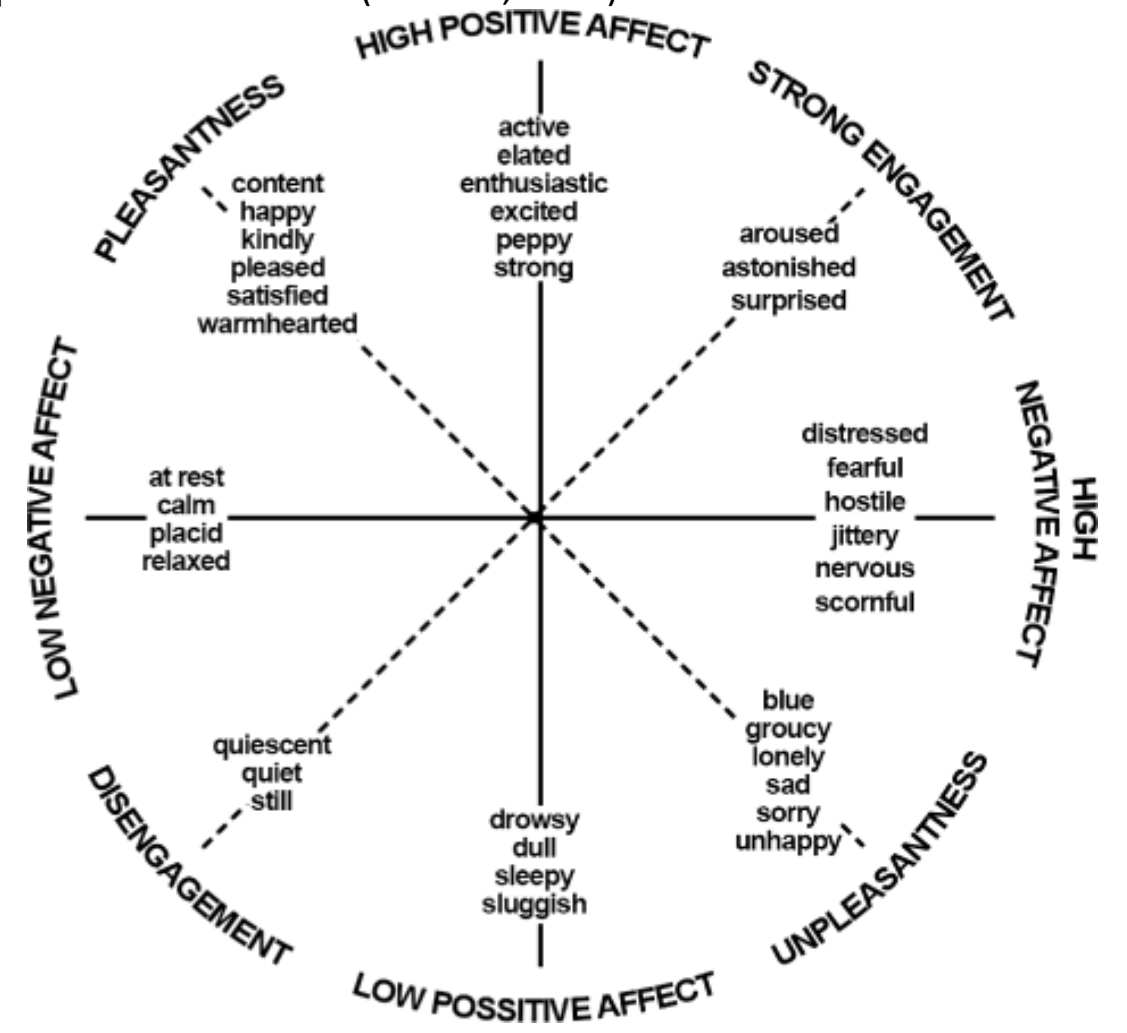

Figure 8: Circumplex Model of Affect (Keller, 1983)

On the other side of coin, negative mood that caused by general factor of subjective distress called as negative affect (Tenney et al., 2016). There is an absence of feelings of pleasure in negative affect and it comprises with a variety of aversive states of mind such as fear (Antunes et al., 2019). Negative affect narrows an individual's attentional focus (Noguchi and Tomoike, 2016). Therefore, any affective state would fall under these two dimensions. Accordingly, the circumplex model of affect will be used as a guideline for the identification of students' affective state in the present research. 


\section{Proposed Conceptual Framework}

The conceptual framework that proposed for the present study is served as a foundation to develop different kawaii designs of pedagogical agents in VLE. The proposed conceptual framework consists of five important layers as shown in figure 9.

Based on the proposed framework, two animated two-dimensional (2D) female pedagogical agents with different kawaii designs are developed to play human instructional roles in VLE. The two different kawaii designs of pedagogical agents are called as kawaii-style pedagogical agent with baby schema and kawaii-style pedagogical agent without baby schema. Both pedagogical agents have different facial appearance with different cuteness ratings based on baby schema features.

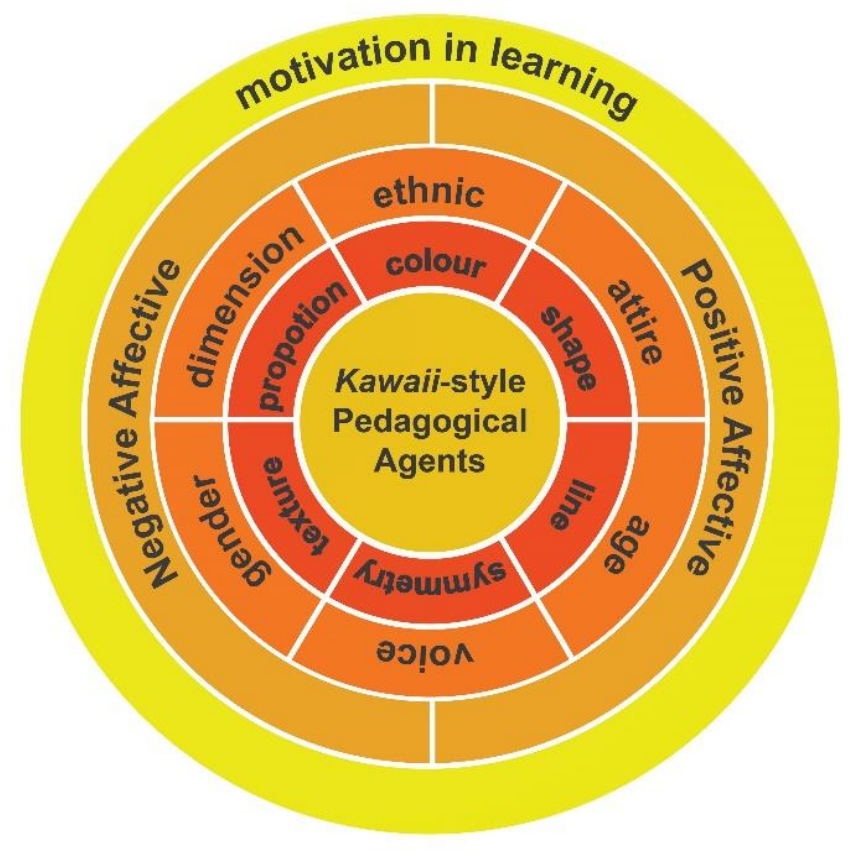

Layer 1 - Two different kawaii designs of pedagogical agents

Layer 2 - Baby schema features

Layer 3 - External factors of target audience

Layer 4 - Positive and negative affective states

Layer 5 - Motivation in learning

Figure 9: Kawaii-style pedagogical agents designs of conceptual framework

The elements of shape and proportion in second layer of the framework determine the extent to which the concept of baby schema is applied to the pedagogical agents. Among the shapes that focus to design the facial characteristics of the pedagogical agents are including eyes, eyebrows, nose, cheeks, lips, chin, forehead, and head. In other words, the kawaii style pedagogical agent with baby schema will be developed to demonstrate babyishness in the facial appearance while kawaii style pedagogical agent without baby schema will be developed to 
represent mature look in the facial appearance. Other than that, other elements such as colour, line, symmetry, and texture also considered in the development of the pedagogical agents.

According to the third layer of the conceptual framework, there are some external factors of target audience that influence the design of pedagogical agents such as attire, age, voice, gender, dimension and ethnic. Students as target audience will be positively affected when each physical features of pedagogical agents match with them. In view of that, the different kawaii designs of pedagogical agents will be designed to match with the target audience.

However, question arises whether the development of different kawaii designs of pedagogical agents will serve as the focal point to stimulate affective state among students? In response to this question, the present study capable to rate students' affective state that will be stimulated during the first look at the designed pedagogical agents. Affective state can be defined as the rising of internal mood that linked to one's attention (White, 2015). Watson and Tellegen (1985) developed a model on the structure of affect. Based on the model, there are two affective state namely positive affect and negative affect. Thus, the fourth layer of the proposed conceptual framework is based on the model of affect which will be used to identify students' affective state towards the designed pedagogical agents.

Adding to this, the stimulated affective state capable to trigger students' motivation in learning in VLE. Basically, there are three types of motivation including intrinsic motivation, extrinsic motivation and amotivation. Intrinsic motivation triggers self-motivation in pursuing learning while extrinsic motivation engages students to continue learning (Li and Lynch, 2016). Amotivation is a state where intrinsic motivation and extrinsic motivation is no longer exist (Yardimci, 2017). Hence, both intrinsic and extrinsic motivation are essential for learning process. In view of that, the designed pedagogical agents will be implemented in VLE to deliver learning materials. Here, students' motivation level will be evaluated to identify whether they are motivated or not throughout the learning process in VLE as shown in fifth layer of the conceptual framework.

\section{Conclusions}

Pedagogical agent is a lifelike character that is presented on a computer screen to support a learning process. Although animated pedagogical agents have been made a good progress in academic research, appropriate facial features of the animated pedagogical agent are yet to be realized for the learning. This is very important to control the affective state of students to influence their learning. This in turn would impact the motivation of students in learning. In view of that, present research focuses on the development of different kawaii designs of pedagogical agents for VLE. The features of the different kawaii design of pedagogical agents are selected in accordance with physical cues that characterizing kawaii with baby schema and kawaii without baby schema. Therefore, the proposed conceptual framework in this study focuses on the design of kawaii-style pedagogical agents in VLE.

\section{Contribution of Study}

Research related to pedagogical agent's design is very essential to elicit maximum effectiveness among students in teaching and learning environment. While the use of pedagogical agent in education is still a growing phenomenon, it is still unclear on the effect of design features of the 
pedagogical agent in learning application. Moreover, the positive impact of cuteness in pedagogical agent has not been shown in education settings. In view of that, an initiative formed to develop different kawaii designs of pedagogical agents to play instructional role in teaching and learning environment. Besides, the introduction of likeable, interesting, attractive, and cute kawaii designs of pedagogical agents capable to elicit more attention from the learner through its feasibility to communicate with students throughout the learning process. The social interaction between the pedagogical agents and students are important stimulus to motivate students to participate actively in learning process. A suitable kawaii design of pedagogical agent will attract students' attention and influence their affective state. Consequently, the students will be very interested to learn independently. Meanwhile, it can also emphasize student centred learning. In addition, the findings of this research capable to provide specific clues in the design of pedagogical agents for education settings. Specifically, the application of facial visual style of pedagogical agent such as colour, shape, appearance, and texture capable to be a set of recommendation towards the development of pedagogical agents. Therefore, this research findings are expected to be a design guideline to the instructional multimedia designer to develop suitable facial design for animated pedagogical agents in virtual learning environment.

\section{Acknowledgment}

Kaliyamah Raman acknowledges the Ministry of Higher Education of Malaysia for awarding the HLP scholarship (Hadiah Latihan Persekutuan) to pursue this research.

\section{References}

Antunes, R., Couto, N., Vitorino, A., Monteiro, D., Marinho, D. A., \& Cid, L. (2019). Physical activity and affect of the elderly: Contribution to the validation of the Positive and Negative Affect Shedule (PANAS) in the Portuguese population. Journal of Human Sport and Exercise, 15(2).

Baylor, A. L., \& Kim, Y. (2005). Simulating Instructional Roles through Pedagogical Agents. International Journal of Artificial Intelligence in Education, 15, 95-115.

Biswas, G., Leelawong, K., Belynne, K., Viswanath, K., Vye, N., Schwartz, D., \& Davis, J. (2004). Incorporating self regulated learning techniques into learning by teaching environments. In Proceedings of the Cognitive Science Society, 26 (26).

Cheok, A. D., \& Fernando, O. N. N. (2012). Kawaii/Cute interactive media. Universal Access in the Information Society, 11(3), 295-309.

Clark, R. C., \& Mayer, R. E. (2003). E-learning and the science of instruction. San Francisco, CA: Pfeiffer.

Dale, J. P. (2016). Cute studies: An emerging field. East Asian Journal of Popular Culture, 2(1), 513.

Detective Pikachu [Image]. (2020). Retrieved from https://en.wikipedia.org/wiki/Detective_Pikachu

Dinçer, S., \& Doğanay, A. (2017). The effects of multiple-pedagogical agents on learners' academic success, motivation, and cognitive load. Computers and Education, 111, 74-100.

Goldberg, B., \& Cannon-Bowers, J. (2015). Feedback source modality effects on training outcomes in a serious game: Pedagogical agents make a difference. Computers in Human Behavior, 52, 1-11. 
Granot, E., Alejandro, T. B., \& Russell, L. T. M. (2014). A socio-marketing analysis of the concept of cute and its consumer culture implications. Journal of Consumer Culture, 14(1), 66-87.

Gulz, A., \& Haake, M. (2006). Design of animated pedagogical agents - A look at their look. International Journal of Human Computer Studies, 64(4), 322-339.

Heidig, S., \& Clarebout, G. (2011). Do pedagogical agents make a difference to student motivation and learning? Educational Research Review 6(1), 27-54.

Hello Kitty [Image]. (2014). Retrieved from https://www.pinclipart.com/downpngs/bJRmbo_permalink-to-clipart-hello-kitty-hellokitty-icon/

Hollands, F. M., \& Tirthali, D. (2014). Resource requirements and costs of developing and delivering MOOCs. International Review of Research in Open and Distance Learning, 15(5), 113-133.

Isen, A. M., \& Reeve, J. (2005). The influence of positive affect on intrinsic and extrinsic motivation: Facilitating enjoyment of play, responsible work behavior, and selfcontrol. Motivation and Emotion, 29(4), 295-323.

Johnson, A. M., Didonato, M. D., \& Reisslein, M. (2013). Animated agents in K-12 engineering outreach: Preferred agent characteristics across age levels. Computers in Human Behavior, 29(4), 1807-1815.

Johnson, W. L., \& Lester, J. C. (2016). Face-to-face interaction with pedagogical agents, twenty years later. International Journal of Artificial Intelligence in Education, 26(1), 25-36.

Keller, J. M. (1983). Motivational design of instruction. Instructional design theories and models: An overview of their current status, 1(1983), 383-434.

Khan, T., Johnston, K., \& Ophoff, J. (2019). The Impact of an Augmented Reality Application on Learning Motivation of Students. Advances in Human-Computer Interaction, 2019.

Kim, Y., \& Baylor, A. L. (2006). Pedagogical agents as learning companions: The role of agent competency and type of interaction. Educational Technology Research and Development, 54(3), 223-243.

Kort, B., Reilly, R., \& Picard, R. W. (2001). An affective model of interplay between emotions and learning: Reengineering educational pedagogy-building a learning companion. In Proceedings - IEEE International Conference on Advanced Learning Technologies, ICALT 2001 (pp. 43-46). IEEE Computer Society.

Lieber-Milo, S., \& Nittono, H. (2019). How the Japanese Term Kawaii Is Perceived Outside of Japan: A Study in Israel. SAGE Open, 9(3).

Li, J., Kizilcec, R., Bailenson, J., \& Ju, W. (2016). Social robots and virtual agents as lecturers for video instruction. Computers in Human Behavior, 55, 1222-1230.

Li, T., \& Lynch, R. (2016). The relationship between motivation for learning and academic achievement among basic and advanced level students studying Chinese as a foreign language in years 3 to 6 at Ascot International School in Bangkok, Thailand. Scholar: Human Sciences, 8(1),

Liu, K., Chen, J. H., \& Chang, K. M. (2019). A study of facial features of American and Japanese cartoon characters. Symmetry, 11(5).

Lorenz, K. (1943). Die angeborenen Formen möglicher Erfahrung. Zeitschrift Für Tierpsychologie, 5(2), 235-409. 
Marcus, A., Kurosu, M., Ma, X., \& Hashizume, A. (2017). Cuteness in Japan. In Springer Series on Cultural Computing (pp. 33-61). Springer.

Martha, A. S. D., \& Santoso, H. B. (2019). The design and impact of the pedagogical agent: A systematic literature review. Journal of Educators Online 16(1).

Mohanty, A. (2016). Affective Pedagogical Agent in E-Learning Environment: A Reflective Analysis. Creative Education, 7(4), 586-595.

Miesler, L., Leder, H., \& Herrmann, A. (2011). Isn't it cute: An evolutionary perspective of babyschema effects in visual product designs. International Journal of Design, 5(3), 17-30.

Nittono, H. (2010). A Behavioral Science Framework for Understanding Kawaii. Proceedings of the Third International Workshop on Kansei, (4), 5-8.

Nittono, H., Fukushima, M., Yano, A., \& Moriya, H. (2012). The Power of Kawaii: Viewing Cute Images Promotes a Careful Behavior and Narrows Attentional Focus. PLOS ONE, 7(9).

Nittono, H. (2016). The two-layer model of 'kawaii': A behavioural science framework for understanding kawaii and cuteness. East Asian Journal of Popular Culture, 2(1), 79-95.

Nittono, H., \& Ihara, N. (2017). Psychophysiological Responses to Kawaii Pictures With or Without Baby Schema. SAGE Open, 7(2).

Noguchi, Y., \& Tomoike, K. (2016). Strongly-motivated positive affects induce faster responses to local than global information of visual stimuli: An approach using large-size Navon letters. Scientific Reports, 6.

Occhi, D. J. (2012). Wobbly aesthetics, performance, and message comparing Japanese kyara with their anthropomorphic forebears. Asian Ethnology, 71(1), 109-132.

Park, S. (2018). Virtual Pedagogical Agents for English Language Teaching and Learning. In The TESOL Encyclopedia of English Language Teaching (pp. 1-9).

Pellitteri, M. (2018). Kawaii Aesthetics from Japan to Europe: Theory of the Japanese "Cute" and Transcultural Adoption of Its Styles in Italian and French Comics Production and Commodified Culture Goods. Arts, 7(3), 24.

Peñalver, J., Salanova, M., Martínez, I. M., \& Schaufeli, W. B. (2019). Happy-productive groups: How positive affect links to performance through social resources. Journal of Positive Psychology, 14(3), 377-392.

Quinn, J. F. (2015). The affect of vision and compassion upon role factors in physician leadership. Frontiers in Psychology, 6:442.

Return of Hanuman [Image]. (2007). Retrieved from http://wallpapers.fansshare.com/gallery/photos/14652645/bal-hanumanmovie/?displaying.

Rodicio, H. G., \& Sánchez, E. (2012). Aids to computer-based multimedia learning: A comparison of human tutoring and computer support. Interactive Learning Environments, 20(5), 423439.

Sherman, G. D., Haidt, J., \& Coan, J. A. (2009). Viewing Cute Images Increases Behavioral Carefulness. Emotion, 9(2), 282-286.

Shiban, Y., Schelhorn, I., Jobst, V., Hornlein, A., Puppe, F., Pauli, P., \& Muhlberger, A. (2015). The appearance effect: Influences of virtual agent features on performance and motivation. Computers in Human Behavior, 49, 5-11.

Sloan, R. (2015). Virtual character design for games and interactive media. New York: A K 
Peters/CRC Press.

Takamatsu, R. (2018). Measuring Affective Responses to Cuteness and Japanese kawaii as a Multidimensional Construct. Current Psychology, 1-13.

Tenney, E. R., Poole, J. M., \& Diener, E. (2016). Does positivity enhance work performance?: Why, when, and what we don't know. Research in Organizational Behavior. JAI Press.

Vicneas, M., \& Zamzuri, A. M. A. (2019). Different realism designs of $2 d$ virtual agents and its arousal effect on students' emotions in learning. International Journal of Engineering and Advanced Technology, 9(1), 5149-5158.

Vicneas, M., \& Zamzuri, A. M. A. (2020). The effect of valence and arousal on virtual agent's designs in quiz based multimedia learning environment. International Journal of Instruction, 13(4), 903-920.

Volkswagen New Beetle [Image]. (2013). Retrieved from https://www.pngegg.com/en/pngtlqcf/download

Yardimci, F., Bektaş, M., Özkütük, N., Muslu, G. K., Gerçeker, G. Ö., \& Başbakkal, Z. (2017). A study of the relationship between the study process, motivation resources, and motivation problems of nursing students in different educational systems. Nurse Education Today, 48, 13-18.

Yu, Y. (2015). The role a motivational agent plays: the effects of a co-learner agent and an expert agent (Master's thesis, University of Twente, Netherlands).

White, W. B. (2015). The relationship between an affective instructional design, children's attitudes toward mathematics, and math learning for kindergarten age children (Master's thesis, East Tennessee State University, United States)

Withrow, S., \& Danner, A. (2007). Character design for graphic novels. Switzerland: RotoVision SA

Wong, S. (2017). Yuru-kyara and Mascot Characters: Cute Aesthetics and the Empathic Effect of Kawaii in Japanese Commercials. 